SENSORY SYSTEMS

\title{
Feeling the warmth
}

the authors
identified a
subset of
C-MC and
C-MHC fibres
that exhibited
ongoing spiking
that was inhibi-
ted by skin
warming

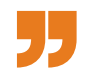

The capacity to perceive small, non-noxious changes in skin temperature enables the regulation of body temperature and appropriate responses to environmental cues. However, our understanding of the sensory coding of temperature, and particularly of innocuous warming, is incomplete. Findings reported by Paricio-Montesinos et al. now suggest that, in mice, the combined activities of warm-activated and warm-inhibited primary sensory afferent neurons are crucial for innocuous warm perception.

Prevailing hypotheses propose that skin temperature changes are conveyed to the CNS via dedicated sensory pathways, or 'labelled lines'. According to this model, the expression of combinations of warm-sensitive or cool-sensitive transient receptor potential (TRP) channels - a number of which have been identified and characterized - endows sensory neurons with specificity for warm or cool stimuli. However, conclusive evidence for this idea, and a full description of the neurons involved, is lacking.
To examine the molecular and cellular mechanisms responsible for encoding warmth, the authors here trained mice on a thermal perception task in which they learned to respond (by licking a sensor) to a temperature 'step' (an increase or decrease in the temperature of a thermal stimulus delivered to their forepaw) to receive a water reward. They discovered that, like humans, mice can perceive small changes in temperature (smaller than $1^{\circ} \mathrm{C}$ ) above or below baseline and distinguish between innocuous warming and cooling.

To identify the populations of sensory neurons involved in the detection of skin warming and cooling, the authors used an ex vivo forepaw skin-nerve preparation. By recording the responses of individual neurons to the application of thermal stimuli to the skin, they could identify and characterize warm-activated and cold-activated neurons.

Almost all of the innocuoustemperature-sensitive neurons that the authors identified were polymodal C fibres that could also be activated by mechanical stimuli and could thus be classified as $\mathrm{C}$-mechanoheat (C-MH), C-mechanoheatcold (C-MHC) or C-mechanocold (C-MC) fibres. Most of these neurons did not fire at baseline temperatures, but increased their firing rates in response to warming (the authors termed these 'warm-excited fibres'). However, when examined at a more physiological temperature $\left(27^{\circ} \mathrm{C}\right.$, corresponding to the author's measurement of mouse forepaw skin temperature), the authors identified a subset of C-MC and C-MHC fibres that exhibited ongoing spiking that was inhibited by skin warming: they termed these 'warm-inhibited afferents'.

To determine which of these neuronal populations drive innocuous temperature perception, the authors compared their activity with the animals' thermal perception at low baseline temperatures $\left(22^{\circ} \mathrm{C}\right)$. At these temperatures, they observed only sparse and weak activation of the warm-excited afferents, but robust responses (increases or decreases of activity) of warm-inhibited afferents to temperature changes. The mice were able to detect the corresponding warming steps robustly and with a short latency, suggesting that the warm-inhibited neurons may play an important role in warm perception.

Next, the authors examined the contribution of known warm-sensitive or cold-responsive TRP channels to innocuous temperature perception. Transgenic mice lacking warmresponsive channels (TRPV1, TRPA1, TRPM3 and TPRM2) were less sensitive to warmth than wild-type mice, but could still detect very small temperature increases (although learning of the perceptual task was delayed in the TRPM2 mutants). This suggested that these channels are involved in, but not essential for, warm perception. By contrast, animals lacking the cold-activated channel TRPM8 could not respond to warming in the perceptual task, suggesting an indispensable role for this receptor in innocuous warmth detection. Forepaw afferent recording demonstrated the specific absence of 'warm-inhibited' fibres in $\operatorname{Tr} p m 8^{-/-}$ mice, further supporting the idea that these fibres are important for warmth perception.

On the basis of these findings, the authors suggest that, rather than using a labelled line strategy, the sensory system may encode innocuous temperature changes via the combination of patterned information from warm-inhibited afferents with that from warm-activated afferents.

Katherine Whalley

ORIGINAL ARTICLE Paricio-Montesinos, R. et al. The sensory coding of warm perception. Neuron https://doi.org/10.1016/j.neuron.2020.02.035 (2020) 